\title{
Analyzing roles of individuals in small-group collaboration processes
}

\author{
Gerry Stahl • Nancy Law $・$ Ulrike Cress $\cdot$ Sten Ludvigsen
}

Published online: 4 November 2014

(C) International Society of the Learning Sciences, Inc. 2014

The papers in this issue present innovative approaches to analyzing the roles of individuals in small-group collaborations supported by computer technologies. In reading these articles, you may find it interesting to consider the ways in which their methods conceptualize the relationship of collaborative group learning to the roles of its individual participants. Taken together, these studies envision and explore a space of possible strategies for analyzing the multi-level phenomena of collaborative learning, sometimes coding utterances of individuals and at other times characterizing group trajectories. They each push the boundaries of CSCL research in various ways. Although they can be read as primarily proposing analytic procedures, they also contribute to theory and technology. Perhaps highlighting their nuanced stances on the issue of unit-of-analysis in probing learning data can help to reveal their contributions to the advance of CSCL as a vision and as a field.

It is often difficult to determine where overall progress is being made in CSCL research and practice. Statistical indicators in comparative reviews tend to be overwhelmed by the diversity of theories and methodologies applied in research and by the variety of pedagogies adopted in practice. In both researcher and teacher communities, there are new participants entering with training in traditional disciplines as well as long-time participants still working within old paradigms. Folk theories derived from common sense linger on and may obscure the visibility of innovations in scientific theory, methodology or pedagogy.

Folk theories of minds and learning still influence classroom practice. According to Bruner (1996) and Bereiter (2002), teachers' pedagogy is often deeply affected by everyday intuitive

G. Stahl $(\triangle)$

Drexel University, Philadelphia, PA, USA

e-mail: Gerry@ijCSCL.org

N. Law

University of Hong Kong, Hong Kong, People's Republic of China

e-mail: Nancy@ijCSCL.org

U. Cress

Knowledge Construction Lab, KMRC-Knowledge Media Research Center, Schleichstr. 6, 72076 Tuebingen, Germany

e-mail: Ulrike@ijCSCL.org

S. Ludvigsen

Medical Informatics, University of Oslo, P.O. Box 1080, Blindern, 0316 Oslo, Norway

e-mail: Sten@ijCSCL.org 
conceptions of how student minds work. This "folk" perspective focuses on the characteristics of the individual learner: In effect, it treats some students as inherently "smart," some as just "dumb." It views students as taking in factual knowledge offered by books or teachers, and committing these to memory so they can retrieve them for tests. Early research on "cooperative" learning - which preceded CSCL — recommended that having students interact could often enhance their individual learning. The smart students would convey their knowledge to the others, and would strengthen their own knowledge by teaching it. Learning in groups was still conceived of as a mental process unfolding inside individual heads. Participants in groups were labeled as having personal attributes like leader, follower, lurker or lazy.

CSCL has always taken a different view of learning. Adopting Piaget's (1929/1990) developmental approach, CSCL views the individual learner as an evolving actor, who changes through interaction with others and with new learning experiences. Following Vygotsky (1930/1978), CSCL explores social, interactional or intersubjective aspects of learning, in which learning takes place initially in the interaction of learners with mentors, teachers or peers, mediated by cultural artifacts. As more recently described by Tomasello (2014), learning by humans (as distinguished, for instance, from apes) is primarily a matter of enculturation, mediated by language and involving distinctively human collective intentionality. Learning takes place in family groupings and cultural institutions, such as schools, museums, libraries, churches and research centers - institutions designed to reproduce and expand our shared cultures.

Even when a learning event takes place in a presumably methodologically isolated mind, that learning - if it is authentic - is motivated by relations with other people, oriented toward future communications with others, dependent on a community's language, founded upon a store of cultural knowledge and probably built upon the words/ideas of others. Thus, an analysis of learning at least has to take into account the influences on the individual of society, communities, culture and other people. CSCL and the learning sciences have recognized this extensively, as they have magnified previous views of cognition through the lenses of sociocultural and dialogical perspectives (Stahl et al. 2014).

However, as we have suggested in the past, it is also possible to conclude that the analysis of learning should consider cognitive processes at the small-group and community levels on a par with those at the individual unit — not just as secondary influences on the individual. Such processes - like collective intentionality, transactive discourse or group agency - might even turn out to be integral to all distinctively human learning. Moreover, such processes might be particularly visible and accessible to educational researchers in CSCL settings. This could lead to a post-cognitive theory of learning in which collaborative learning is not just a niche distraction from individual learning, but rather a necessary foundation for it.

We have proposed in recent editorials in ijCSCL that a primary focus of CSCL research today should be on the relationship among processes at different units of analysis, such as individual student, small group and classroom or community (Stahl 2012, 2013a). We have illustrated possible ways of relating multiple levels in our own publications (e.g., Arnseth and Ludvigsen 2006; Cress 2008; Cress and Kimmerle 2008; Law et al. 2012; Stahl 2013b, 2015). The papers in this issue take concrete preliminary steps in such directions.

\section{Joint attention in a dyad}

Joint attention is the sine qua non of collaboration. How can people work together if they are not oriented to the same thing? Tomasello's (2014) comparative research with primates shows that joint attention is a peculiarly human skill, nurtured from infancy, as illustrated in 
Vygotsky's analysis of the emergence of the intersubjective pointing gesture in the motherbaby dyad (1930/1978, p.56). Shared intentionality makes possible intersubjective meaning making in human groups.

In face-to-face interaction, a variety of deictic gestures, gazes and bodily orientations help to direct attention (Evans et al. 2011). In online contexts, an array of subtle linguistic indexical referencing practices dominate (Zemel and Koschmann 2013). In addition, computer environments can provide technical supports for coordinating attention (Mühlpfordt and Wessner 2009).

In their article below on shared sensing of gaze, Bertrand Schneider and Roy Pea analyze the impact of an eye-tracking tool to enhance joint attention. In a contribution to ijCSCL last year, they had showed how displaying the gaze of a partner helped a dyad to collaborate and learn (Schneider and Pea 2013). Now, they develop representations of eye-gaze coordination to predict the quality of collaboration.

They devise a task that requires extensive comparison of visual features at multiple locations on the screen, requiring complex patterns of eye movements. To collaborate on the task, it helps subjects if they can follow each other's gaze - as technologically supported in the experimental condition. The authors analyze the pros and cons for researchers of several representations of the dyads' eye movements, tracking both individual movements and coordinated dyad trajectories. Given the nature of the task, it is often possible to surmise when a subject following the gaze of the partner understands the partner's intention, seeing the meaning of shifts in gaze.

While this study is carefully confined to its research questions, it also raises the possibility of using eye-tracking evidence of joint attention to complement the analysis of other dimensions of collaboration, such as discourse. Can awareness of gaze by participants in online collaboration reduce the frequency of misunderstandings and the necessity of repair moves? Can it provide researchers with evidence to resolve interpretive ambiguities in the often-terse format of online discourse? If so, the technology and the analytic representations could help to counter certain problematic circumstances in CSCL practice and research.

In terms of the relationship of the individual and group units of analysis, the approach taken here demonstrates how they are intimately intertwined. Joint attention is a group phenomenon. It involves both members of the dyad not only gazing at the same object, but gazing with the same intent as each other (for instance to compare the focal object in a certain way with a specific other object in the task). Each member believes that the other intends it that way, and knows that they are gazing together, relating to a shared focal object, taken as the same. The mutuality of intersubjective intentionality is not reducible to either individual's mental representations, but consists in a shared meaning making. However, in addition to being often tightly bound within the dyad, the participants also go off individually in many moments. In fact, the study concludes that the quality of the collaboration is related to the ratio of group to individual activity. Like all group processes, there is an ebb and flow of group cohesion. CSCL approaches and environments can foster higher levels of collaboration by understanding how individual and group activities feed into and constitute each other.

\section{Leadership as interactional}

Leadership is another aspect of collaborative learning undergoing increasingly nuanced analysis. Emma M. Mercier, Steven E. Higgins and Laura da Costa examine the work of student groups using multi-touch tablets. The authors build on their previous study of pedagogies related to this technology (Higgins et al. 2011). They view the group as the 
cognitive unit and see leadership as a form of interaction within the group. For instance, if an individual makes a proposal that is ignored and not taken up by other members of the group, then that action is classified as a "failed bid" at leadership. It does not count as leadership. A leadership move involves a bid being taken up by others (see Stahl 2006, Ch.21), so it takes place at the interactional group unit of analysis.

The research reported here analyzes leadership moves as attempts to push the group forward by addressing either relational or content matters. Relational issues of group organization include concerns such as turn control, while content issues address intellectual aspects of the task activity, such as idea management and development. Collaborative learning has long been seen within CSCL to include both a content realm or "joint problem space" (Teasley and Roschelle 1993) and a group relational dimension (Barron 2003). In this paper, the authors present an analytic approach for studying leadership as a group effort encompassing both these dimensions.

This analysis moves us far from the idea that leaders are just born that way. It sees leadership as emergent from group interaction and distributed among group members. However, beyond that, it examines the way that leadership moves drive the group process and the collaborative knowledge building forward, and how those moves may be made by different individuals as a result of the sequential group dynamic.

\section{Roles as group process}

Leadership has frequently been conceptualized in CSCL practice as a role for a particular individual within a group. Often, it is suggested that a teacher assign one student in each small group to take on some kind of leadership role, while another student might be assigned a different role, such as timekeeper (Pozzi 2011; Schellens et al. 2007). In current research, we now see roles being conceptualized as group processes, addressing multiple distinct facets of collaboration and being naturally distributed across the group, i.e., consisting of interactions between group members.

The next article, by H. Smith Risser and SueAnn Bottoms, looks at the variety of social roles associated with blogging, including forms of leadership. It uses a cluster-analysis method based on social-network analysis, rather than coding, to determine categories of roles, to detect what roles individuals have in blogging communities and to track how those roles shift. The set of these roles is quite dependent upon the specific blog technology. Blogs may, for instance, involve different types of hyperlinks: e.g., blog-rolls, citation links and comment links. Individuals' prominence in the community depends upon their participation in these different kinds of links.

The cluster analysis results in five distinct categories of social roles in the blog community. Although the authors name the five clusters with terms derived from the theory of Lave and Wenger (1991), the categories are actually computed from network analysis of blogging communities. The clusters are called: relative newcomers, inbound participants, peripheral members, full participants and celebrities. The names indicate differing levels of recognition and participation typical in each cluster. In folk theories, celebrity status was assumed to be an attribute of an individual. Here it is derived from the complex network structure of interaction across an active blog community. Roles are group-level characteristics of social structures.

\section{Uncertainty in collaborative learning}

Leadership in collaborative learning is - perhaps counter-intuitively-often expressed in a mode of uncertainty. In the final article of this year's volume, Michelle E. Jordan, An-Chih 
Janne Cheng, Diane Schallert, Kwangok Song, SoonAh Lee and Yangjoo Park explore the frequent co-occurrence of expressions of uncertainty with effective learning interactions. They document that learning is often expressed with terms of uncertainty and they explore some reasons for this. The tasks undertaken by their subjects involved building knowledge of sophisticated and nuanced ideas, making certainty elusive. However, even more generally, learning by definition involves something new, about which one is not already knowledgeable, and thus often feels tentative. By using hedges, hypotheticals and questions, people can open up a space for safe exploration and provisional statements. Furthermore, by writing ideas online for others, expressions of learning can be treated as dialogical acts, conducive to learning through negotiation over time with others, rather than requiring absolute certitude from the start by an individual.

The notion of using questioning modes of expression rather than propositional pronouncements led the authors to propose an alternative approach to their focus on individual learning and individual certainty. They suggest exploring the possibility that "uncertainty co-occurs with learning, but may not coincide with learning in the same message or by the person expressing the uncertainty. Such an analysis ... would require a more dynamic sequential analysis." This would shift the data for examination from the individual to the interactional group unit of analysis. It would view the questioning or uncertain phrasing as an elicitation by the writer for a reader to affirm or modify the proposed expression of learning. The learning would then be a fully dialogical or group act, facilitated by uncertainty expressions that call for confirmatory responses or discussion.

As in the other papers, we see here how a collaborative small group takes action: one member proposes something and the others adopt it (or not). The actions - paying attention, adopting a social role, providing leadership, expressing learning - consist of inter-actions among individual members, resulting in something that cannot be attributed to any one of the individuals or analyzed at the individual unit of analysis because it is an interaction within the group. It might be useful to view such actions as components of group agency (Damsa 2014). Agency is a temporal undertaking (Emirbayer and Mische 1998), including deciding what to do in the future, managing the effort now and evaluating the result of past action. Group agency requires joint attention, coordinated action and intersubjective intentionality (shared meaning making). It can involve leadership, social roles and uncertainty. It can be analyzed in the behavior of individuals and of collaborative groups. It can be supported in CSCL environments. The four studies in this issue suggest a methodological orientation toward group agency, which could lead CSCL beyond traditional theories of individual rational decision making and mental representation to the foundations of collaborative learning in the group interaction.

\section{CSCL 2015}

We look forward to seeing you in June at CSCL 2015 (isls.org/cscl2015) in Gothenburg, Sweden. Opportunities and challenges of CSCL today will be displayed and discussed there.

\section{References}

Arnseth, H. C., \& Ludvigsen, S. (2006). Approaching institutional contexts: Systemic versus dialogic research in CSCL. International Journal of Computer-Supported Collaborative Learning, 1(2), 167-185.

Barron, B. (2003). When smart groups fail. The Journal of the Learning Sciences, 12(3), 307-359. 
Bereiter, C. (2002). Education and mind in the knowledge age. Hillsdale: Lawrence Erlbaum Associates.

Bruner, J. (1996). The culture of education. Cambridge: Harvard University Press.

Cress, U. (2008). The need for considering multilevel analysis in CSCL research: An appeal for the use of more advanced statistical methods. International Journal of Computer-Supported Collaborative Learning, 3(1), 69-84.

Cress, U., \& Kimmerle, J. (2008). A systemic and cognitive view on collaborative knowledge building with wikis. International Journal of Computer-Supported Collaborative Learning, 3(2), 105-122.

Damsa, C. (2014). The multi-layered nature of small-group learning: Productive interactions in object-oriented collaboration. International Journal of Computer-Supported Collaborative Learning, 9(3), 247-281.

Emirbayer, M., \& Mische, A. (1998). What is agency? American Journal of Sociology, 103(4), 962-1023.

Evans, M. A., Feenstra, E., Ryon, E., \& McNeill, D. (2011). A multimodal approach to coding discourse: Collaboration, distributed cognition, and geometric reasoning. International Journal of Computer-Supported Collaborative Learning, 6(2), 253-278.

Higgins, S. E., Mercier, E., Burd, E., \& Hatch, A. (2011). Multi-touch tables and the relationship with collaborative classroom pedagogies: A synthetic review. International Journal of Computer-Supported Collaborative Learning, 6(4), 515-538.

Lave, J., \& Wenger, E. (1991). Situated learning: Legitimate peripheral participation. Cambridge: Cambridge University Press.

Law, N., Yuen, J., \& Tse, H. (2012). A teacher's journey in knowledge building pedagogy. In the Proceedings of the 10th International Conference of the Learning Sciences (ICLS 2012). J. v. Aalst, K. Thompson, M. J. Jacobson \& P. Reimann. Sydney, Australia. Proceedings pp. 212-219. ISLS.

Mühlpfordt, M., \& Wessner, M. (2009). The integration of dual-interaction spaces. In G. Stahl (Ed.), Studying virtual math teams. (ch. 15, pp. 281-293). New York: Springer. Web: http://GerryStahl.net/vmt/book/15.pdf.

Piaget, J. (1929/1990). The child's conception of the world. New York: Littlefield Adams.

Pozzi, F. (2011). The impact of scripted roles on online collaborative learning processes. International Journal of Computer-Supported Collaborative Learning, 6(3), 471-484.

Schellens, T., Van Keer, H., De Wever, B., \& Valcke, M. (2007). Scripting by assigning roles: Does it improve knowledge construction in asynchronous discussion groups? International Journal of Computer-Supported Collaborative Learning, 2(2-3), 225-246.

Schneider, B., \& Pea, R. (2013). Real-time mutual gaze perception enhances collaborative learning and collaboration quality. International Journal of Computer-Supported Collaborative Learning, 8(4), 375-397.

Stahl, G. (2006). Group cognition: Computer support for building collaborative knowledge. Cambridge, MA: MIT Press. Web: http://GerryStahl.net/elibrary/gc.

Stahl, G. (2012). Traversing planes of learning. International Journal of Computer-Supported Collaborative Learning, 7(4), 467-473.

Stahl, G. (2013a). Learning across levels. International Journal of Computer-Supported Collaborative Learning, $8(1), 1-12$.

Stahl, G. (2013b). Translating Euclid: Designing a human-centered mathematics (paperback \& ebook ed.). San Rafael: Morgan \& Claypool Publishers. 221 pages. Web: http://GerryStahl.net/elibrary/euclid.

Stahl, G. (2015). The group as paradigmatic unit of analysis: The contested relationship of CSCL to the learning sciences. In M. Evans, M. Packer \& K. Sawyer (Eds.), The learning sciences: Mapping the terrain. Cambridge: Cambridge University Press. Web: http://GerryStahl.net/pub/ls.pdf.

Stahl, G., Cress, U., Ludvigsen, S., \& Law, N. (2014). Dialogic foundations of CSCL. International Journal of Computer-Supported Collaborative Learning, 9(2), 1-9.

Teasley, S. D., \& Roschelle, J. (1993). Constructing a joint problem space: The computer as a tool for sharing knowledge. In S. P. Lajoie \& S. J. Derry (Eds.), Computers as cognitive tools (pp. 229-258). Mahwah: Lawrence Erlbaum Associates, Inc.

Tomasello, M. (2014). A natural history of human thinking. Cambridge: MA: Harvard University Press.

Vygotsky, L. (1930/1978). Mind in society. Cambridge: Harvard University Press.

Zemel, A., \& Koschmann, T. (2013). Recalibrating reference within a dual-space interaction environment. International Journal of Computer-Supported Collaborative Learning, 8(1), 65-87. 